Erratum

\title{
Erratum à « Borréliose de Lyme et autres maladies vectorielles à tiques. Recommandations des sociétés savantes françaises » [Med. Mal. Infect. 49 (2019) 296-317]
}

\section{Erratum to "Lyme borreliosis and other tick-borne diseases. Guidelines from the French scientific societies" [Med. Mal. Infect. 49 (2019) 296-317]}

\author{
X. Gocko ${ }^{a}$, C. Lenormand ${ }^{b}$, C. Lemogne ${ }^{c}$, K. Bouiller $^{\mathrm{d}}$, J.-F. Gehanno ${ }^{\mathrm{e}}$, C. Rabaud $^{\mathrm{f}}$,
} S. Perrot ${ }^{g}$, C. Eldin ${ }^{\mathrm{h}}$, T. de Broucker ${ }^{\mathrm{i}}$, F. Roblot ${ }^{\mathrm{j}}$, J. Toubiana $^{\mathrm{k}}$, F. Sellal ${ }^{\mathrm{l}}$, F. Vuillemet ${ }^{\mathrm{l}}$, C. Sordet ${ }^{\mathrm{m}}$, B. Fantin ${ }^{\mathrm{n}}$, G. Lina ${ }^{\mathrm{p}}$, C. Sobas ${ }^{\mathrm{N}}$, B. Jaulhac ${ }^{\mathrm{p}}$, J. Figoni $^{\mathrm{q}, \mathrm{r}}$, C. Chirouze $^{\mathrm{d}}$, Y. Hansmann ${ }^{\mathrm{s}}$, V. Hentgen ${ }^{\mathrm{t}}$, E. Caumes ${ }^{\mathrm{u}}$, M. Dieudonné ${ }^{\mathrm{v}}$, O. Picone ${ }^{\mathrm{w}}$, B. Bodaghi ${ }^{\mathrm{x}}$, J.-P. Gangneux ${ }^{\mathrm{y}}$, B. Degeilh ${ }^{\mathrm{y}}$, H. Partouche ${ }^{\mathrm{z}}$, A. Saunier ${ }^{\mathrm{a}}$, A. Sotto ${ }^{\mathrm{ab}}$, A. Raffetin ${ }^{\mathrm{ac}}$, J.-J. Monsuez ${ }^{\text {ad }}$, C. Michel ${ }^{\text {ae }}$, N. Boulanger ${ }^{\mathrm{P}}$, P. Cathebras ${ }^{\text {af }}$, P. Tattevin ${ }^{\text {ag, }}{ }^{*}$, endorsed by the following scientific societies ${ }^{1}$

a Département de médecine générale, faculté de médecine, Saint-Étienne, France

b Dermatologie, hôpitaux universitaires de Strasbourg et faculté de médecine, université de Strasbourg, France

c Psychiatrie, hôpital européen Georges-Pompidou, AP-HP.5, Inserm U1266, université Paris Descartes, Paris, France

${ }^{\mathrm{d}}$ Maladies infectieuses et tropicales, centre hospitalo-universitaire, UMR CNRS 6249 université Bourgogne Franche Comté, Besançon, France

e Médecine du travail, centre hospitalo-universitaire, Rouen, France

${ }^{\mathrm{f}}$ Maladies infectieuses et tropicales, centre hospitalo-universitaire, Nancy, France

g Centre d'étude et de traitement de la douleur, hôpital Cochin, Paris, France

${ }^{\mathrm{h}}$ Maladies infectieuses et tropicales, IHU Méditerranée infection, centre hospitalo-universitaire Timone, Marseille, France

i Neurologie, hôpital Delafontaine, Saint-Denis, France

$\mathrm{j}$ Inserm U1070, Maladies infectieuses et tropicales, centre hospitalo-universitaire, Poitiers, France

${ }^{\mathrm{k}}$ Service de pédiatrie générale et maladies infectieuses, hôpital Necker-Enfants malades, AP-HP, Paris, France

${ }^{1}$ Département de neurologie, hôpitaux Civil, Colmar, France

m Rhumatologie, centre hospitalo-universitaire, Strasbourg, France

${ }^{n}$ Médecine interne, hôpital Beaujon, université Paris Diderot, Inserm UMR 1137 IAME, Clichy, France

${ }^{\circ}$ Microbiologie, centre hospitalo-universitaire, Lyon, France

${ }^{\mathrm{P}}$ Laboratoire de bactériologie et CNR des Borrelia, faculté de médecine et centre hospitalo-universitaire, Strasbourg, France

${ }^{\mathrm{q}}$ Maladies infectieuses et tropicales, hôpital Avicenne, Bobigny, France

${ }^{\mathrm{r}}$ Santé publique France, St.-Maurice, France

${ }^{s}$ Maladies infectieuses et tropicales, centre hospitalo-universitaire, Strasbourg, France

${ }^{\mathrm{t}}$ Pédiatrie, centre hospitalier, Versailles, France

"Maladies infectieuses et tropicales, hôpital La Pitié-Salpêtrière, Paris, France

DOI de l'article original : https://doi.org/10.1016/j.medmal.2019.05.006.

* Auteur correspondant. Service des maladies infectieuses et réanimation médicale, CHU Pontchaillou, 35033 Rennes cedex, France.

Adresse e-mail : pierre.tattevin@chu-rennes.fr (P. Tattevin).

${ }^{1}$ Société française de dermatologie (SFD), Société française de rhumatologie (SFR), Fédération française de neurologie (FFN), Société française de neurologie (SFN), Collège

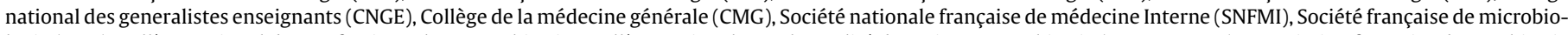

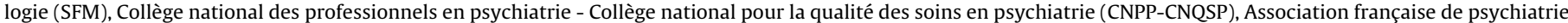

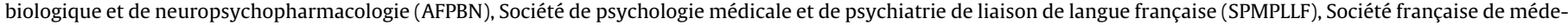



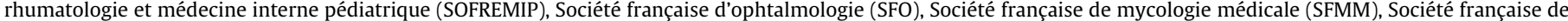

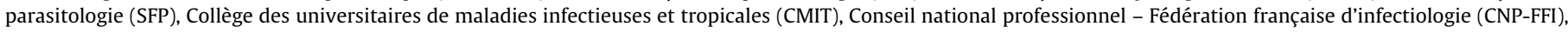

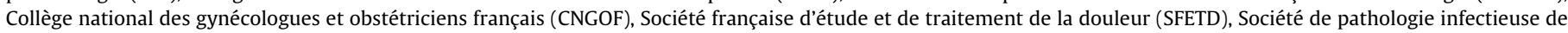
langue française (SPILF). 
${ }^{v}$ Centre Max-Weber, CNRS, université Lyon 2, Lyon, France

${ }^{\mathrm{w}}$ Maternité Louis-Mourier, 92700 Colombes, France

x Ophtalmologie, hôpital La Pitié-Salpêtrière, 75013 Paris, France

${ }^{y}$ Laboratoire de parasitologie-mycologie, UMR S 1085 Irset, université Rennes1-Inserm-EHESP, centre hospitalo-universitaire, 35000 Rennes, France

${ }^{z}$ Cabinet de médecine générale, 93400 Saint-Ouen, département de médecine générale, faculté de médecine, université Paris Descartes, 75006 Paris, France

aa Médecine interne et maladies infectieuses, centre hospitalier, 24750 Périgueux, France

${ }^{\mathrm{ab}}$ Maladies infectieuses et tropicales, centre hospitalo-universitaire, 30000 Nîmes, France

${ }^{a c}$ Maladies infectieuses et tropicales, centre hospitalier intercommunal, 94190 Villeneuve-St-Georges, France

${ }^{\text {ad }}$ Cardiologie, hôpital René-Muret, 93270 Sevran, France

ae Médecine générale, 67000 Strasbourg, France

af Médecine interne, hôpital Nord, centre hospitalo-universitaire, 42000 Saint-Étienne, France

ag Maladies infectieuses et reanimation médicale, hôpital Pontchaillou, centre hospitalo-universitaire, 35000 Rennes, France

L'article " Lyme borreliosis and other tick-borne diseases. Guidelines from the French scientific societies " paru dans le numéro 49/5 comportait une liste de co-auteurs non exhaustive, il manquait le nom de $M$. O. Picone.

Nous prions nos lecteurs de nous en excuser.

Nous vous présentons ci-dessous la liste telle qu'elle aurait dû apparaître.
X. Gocko, C. Lenormand, C. Lemogne, K. Bouiller, J.-F. Gehanno, C. Rabaud, S. Perrot, C. Eldin, T. de Broucker, F. Roblot, J. Toubiana, F. Sella 1, F. Vuillemet, C. Sordet, B. Fantin, G. Lina, C. Sobas, B. Jaulhac, J. Figoni,r, C. Chirouze, Y. Hansmann, V. Hentgen, E. Caumes, M. Dieudonné, O. Picone, B. Bodaghi, J.-P. Gangneux, B. Degeilh, H. Partouche, A. Saunier, A. Sotto, A. Raffetin, J.-J. Monsuez, C. Michel, N. Boulanger, P. Cathebras, P. Tattevin*, endorsed by the following scientific societies.

DOI de l'article original : https://doi.org/10.1016/j.medmal.2019.05.006. 